\title{
Papers
}

\section{Modelling the cost effectiveness of interferon beta and glatiramer acetate in the management of multiple sclerosis}

\author{
Jim Chilcott, Chris McCabe, Paul Tappenden, Anthony O'Hagan, Nicola J Cooper, Keith Abrams, \\ Karl Claxton on behalf of the Cost Effectiveness of Multiple Sclerosis Therapies Study Group
}

\begin{abstract}
Objective To evaluate the cost effectiveness of four disease modifying treatments (interferon betas and glatiramer acetate) for relapsing remitting and secondary progressive multiple sclerosis in the United Kingdom.

Design Modelling cost effectiveness.

Setting UK NHS.

Participants Patients with relapsing remitting multiple sclerosis and secondary progressive multiple sclerosis.

Main outcome measures Cost per quality adjusted life year gained.

Results The base case cost per quality adjusted life year gained by using any of the four treatments ranged from $£ 42000$ (\$66 469; €61 630) to $£ 98000$ based on efficacy information in the public domain. Uncertainty analysis suggests that the probability of any of these treatments having a cost effectiveness better than $£ 20000$ at 20 years is below $20 \%$. The key determinants of cost effectiveness were the time horizon, the progression of patients after stopping treatment, differential discount rates, and the price of the treatments.

Conclusions Cost effectiveness varied markedly between the interventions. Uncertainty around point estimates was substantial. This uncertainty could be reduced by conducting research on the true magnitude of the effect of these drugs, the progression of patients after stopping treatment, the costs of care, and the quality of life of the patients. Price was the key modifiable determinant of the cost effectiveness of these treatments.
\end{abstract}

\section{Introduction}

Multiple sclerosis is a demyelinating disease of the central nervous system. ${ }^{1}$ It is the most common cause of neurological disability in young adults. Four types of disease have been defined: benign or stable, relapsing remitting, secondary progressive, and primary progressive multiple sclerosis. We evaluated the cost effectiveness of four drugs for multiple sclerosis. We assessed the effect of each drug against conventional management.

Until the 1990 s there was no disease modifying treatment for multiple sclerosis. Management of the disease consisted of symptom control, physiotherapy, psychiatric and social support, and disability aids. At present the only disease modifying therapies available for relapsing remitting and secondary progressive multiple sclerosis are interferon beta and glatiramer acetate, which reduce the number of relapses and the rate at which patients progress through the disease. Up to 63000 people in England and Wales have multiple sclerosis, but this estimate may be low. Up to $30 \%$ of the population with multiple sclerosis could be eligible for treatment with these drugs. ${ }^{2}$

Four disease modifying therapies are licensed for the treatment of relapsing remitting multiple sclerosis in the United Kingdom: interferon beta-1a (6 MIU/week; Avonex, Biogen), interferon beta-1b (8 MIU/week; Betaferon, Schering Health), glatiramer acetate (20 mg/week; Copaxone, Teva), interferon beta-1a (22 $\mu \mathrm{g}$ /week; Rebif, Serono), and interferon beta-1a (44 $\mu \mathrm{g}$ /week; Rebif). Interferon beta- $1 \mathrm{~b}$ is the only drug licensed for the treatment of secondary progressive multiple sclerosis in the United Kingdom. The Association of British Neurologists has set out eligibility criteria for treatment with interferon beta and glatiramer acetate (box 1). ${ }^{3}$

Many attempts have been made to estimate the cost effectiveness of these treatments for multiple sclerosis. Analyses have produced cost effectiveness estimates ranging from over $£ 1 \mathrm{~m}$ per quality adjusted life year (QALY) gained to cost saving. ${ }^{4-9}$ Owing to major flaws in the modelling of the clinical course of multiple sclerosis, efficacy, discontinuation of treatment, mortality, and the analysis of uncertainty, none of these estimates can be considered robust. ${ }^{8}$ The Cost Effectiveness of Multiple Sclerosis Therapies Study Group was commissioned by the National Institute for Clinical Excellence to undertake this economic assessment in

Box 1: Association of British Neurologists eligibility criteria for treatment with interferon beta and glatiramer acetate in multiple sclerosis

- Able to walk independently

- At least two major relapses in the past two years

- Aged at least 18 years

- No contraindications

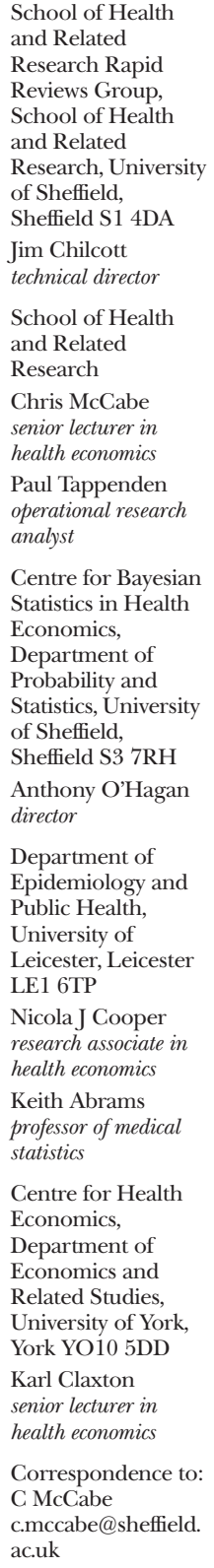

School of Health and Related Research Rapid Reviews Group, School of Health and Related Research, University of Sheffield, Sheffield S1 4DA Jim Chilcott technical director School of Health and Related Research

Chris McCabe senior lecturer in health economics Paul Tappenden operational research analyst

Centre for Bayesian Statistics in Health

Economics,

Department of

Probability and

Statistics, University

of Sheffield,

Sheffield S3 7RH

Anthony O'Hagan director

Department of Epidemiology and Public Health, University of Leicester, Leicester LE1 6TP

Nicola J Cooper research associate in health economics

Keith Abrams professor of medical statistics

Centre for Health Economics, Department of Economics and Related Studies, University of York, York YO10 5DD

Karl Claxton senior lecturer in health economics

Correspondence to: C McCabe c.mccabe@sheffield. ac.uk

bmj.com 2003;326:522 
Box 2: Kurtzke expanded disability status scale

- 0 -normal neurological examination

- 1.0-3.5-neurological impairments that are likely to have limited impact on the activities of daily living

- 4.0-5.5-ambulatory limitations for distances up to $500 \mathrm{~m}$

- 6.0-9.5-require mobility aids. As disease progresses, patients may require a wheelchair and eventually become bedridden

- 10-death due to multiple sclerosis

consultation with all stakeholders to its appraisals process, using the best available evidence.

\section{Methods}

We constructed a model to simulate the clinical course of the disease. Health states were defined according to the Kurtzke expanded disability status scale (box 2). ${ }^{10}$ This scale measures disease progression in terms of impairment and disability. We modelled disability status from 0 to 10 for relapsing remitting multiple sclerosis and from 2 to 10 for secondary progressive multiple sclerosis. ${ }^{10}$

We assessed the clinical course of the disease, costs, and utilities with and without treatment over 20 years. Improvements on the scale are not possible in our model.

Our model used an annual cycle length. Figure 1 shows the transitions between health states that are possible during each cycle. Patients can remain in their current health state, progress one or more states, die, transit to a secondary progressive health state, or stop treatment. Patients who stop treatment progress according to the transition rates for conventional management, retaining the benefits of treatment achieved up to the point of stopping treatment. ${ }^{11}$

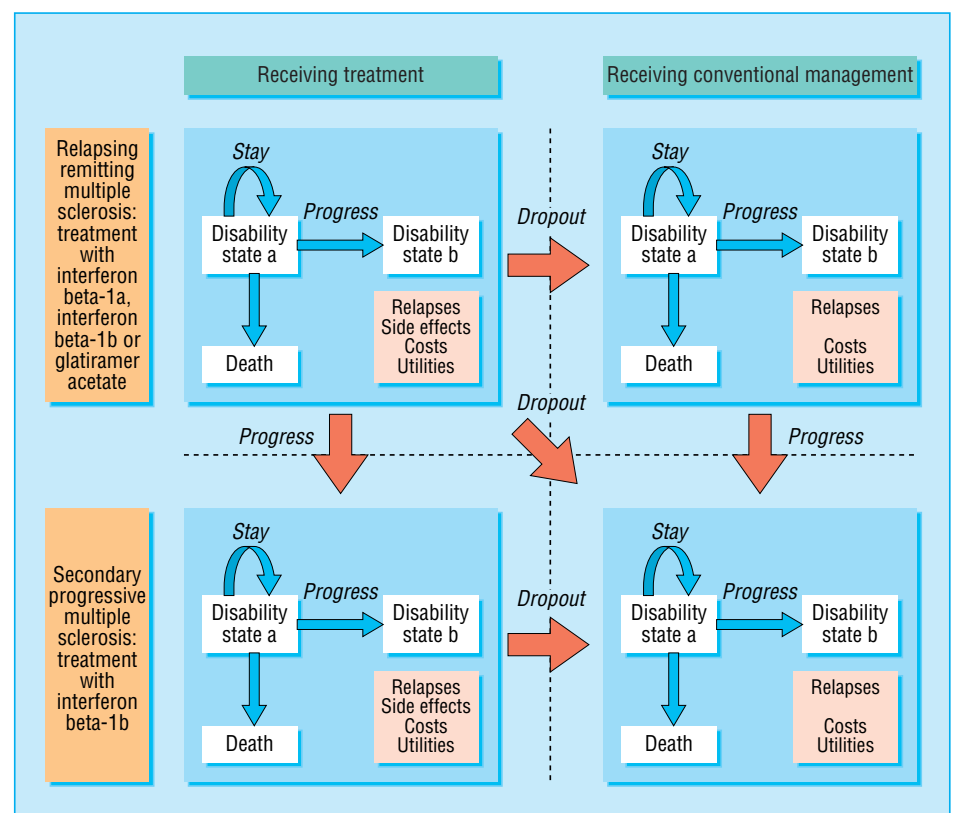

Fig 1 Model of transitions between health states of patients with relapsing remitting or secondary progressive multiple sclerosis

\section{Progression and relapses}

We derived disease progression rates under conventional management from a large study conducted over 25 years, based on a sample population of more than 1000 patients with essentially untreated multiple sclerosis. Enrolment to this study ended in $1984 .{ }^{11}{ }^{12} \mathrm{We}$ excluded patients who were not eligible for treatment according to licensed indications and the Association of British Neurologists guidelines (see box 1). ${ }^{3}$

We derived relapse rates from a cohort study that reported relapses for each year since diagnosis rather than for each disability state. ${ }^{13}$ We used the transition matrices for disease progression under conventional management to calculate the expected time since diagnosis for each disability state. We then used these to estimate the probability of relapse for each disability state in the model.

\section{Effectiveness}

We derived relative risks of relapse and disease progression from four published trials of interferon beta and one trial of glatiramer acetate (table 1). ${ }^{14-18}$ In addition we were able to reanalyse trial data held as commercial in-confidence for three of the products to produce alternate relative risks for relapse and disease progression. ${ }^{19-20}$ We made all the results for efficacy and cost effectiveness available to the appraisal committee of the National Institute for Clinical Excellence; only those derived from publicly available efficacy data and those in-confidence results approved for publication are reproduced here in full.

\section{Costs}

We estimated the costs of managing disability and relapse in each health state from a UK based patient dataset comprising 622 records. $^{21}$ We excluded 244 records because the patient had primary progressive multiple sclerosis, benign multiple sclerosis, or there were no data on disability status. Management of patients in state 3.0 was around twice as expensive as management in state 1.0, management of patients in state 7.0 was around 10 times as expensive as management in state 3.0, and management in states 9.0 and 9.5 was twice as expensive as management in state 7.0.

The costs of interferon beta-1a (6 MIU/week 22 $\mu \mathrm{g} /$ week, and $44 \mu \mathrm{g} /$ week) and glatiramer acetate (20 $\mathrm{mg}$ /week) were taken from the British National Formulary according to licensed dosages. ${ }^{22}$ The cost of 8 $\mathrm{MIU} /$ week interferon beta-1b was obtained from the manufacturer.

\section{Health outcomes used in model}

We obtained the quality of life for each disability state and the disutility of relapses from a dataset of 1552 patients with multiple sclerosis in the United Kingdom. The Multiple Sclerosis Research Trust made this dataset available to the National Institute for Clinical Excellence for the purposes of the appraisal. ${ }^{23}$ We used data from patients with relapsing remitting multiple sclerosis and secondary progressive multiple sclerosis $(\mathrm{n}=780)$. We used a generalised linear regression model of EQ-5D single index score as a function of type of multiple sclerosis, disability status, and relapse status to estimate the utility for each disability state and the disutility of relapse. We made the results on utility available to the appraisal committee of the National Institute for Clinical Excellence, however they are com- 
Table 1 Details of published trials of interferon betas and glatiramer acetate in multiple sclerosis

\begin{tabular}{lccc}
$\begin{array}{l}\text { Trial } \\
\text { Interferon beta Multiple Sclerosis Study Group }\end{array}$ & No of patients & Type of disease & $\begin{array}{c}\text { Intervention } \\
\text { Duration of follow } \\
\text { up (years) }\end{array}$ \\
\hline PRISMS $^{15}$ & 372 & Relapsing remitting & $\begin{array}{c}\text { Placebo versus 1.6 MIU/week or } 8 \mathrm{MIU} / \text { week } \\
\text { interferon beta-1b }\end{array}$ \\
\hline Jacobs et al $^{16}$ & 560 & Relapsing remitting & $\begin{array}{c}\text { Placebo versus } 22 \mu \mathrm{mg} / \text { week or } 44 \mu \mathrm{g} / \text { week } \\
\text { interferon beta-1a }\end{array}$ \\
\hline Johnson et al $^{17}$ & & Relapsing remitting & Placebo versus 6 MIU/week interferon beta-1a \\
\hline European Study Group $^{18}$ & 301 & Relapsing remitting & Placebo versus $20 \mu \mathrm{g}$ glatiramer acetate \\
\hline
\end{tabular}

mercial in-confidence and are not reproduced here. We estimated the difference between state 0 and state 3.0 to represent around a $30 \%$ reduction in the patient's quality of life. We found a similar reduction in quality of life from state 3.0 to state 7.0 ; we estimated that states 9.0 and 9.5 were worse than death-that is, the quality of life was less than zero.

\section{Discounting}

Good practice requires that costs and benefits that occur in the future are given less weight than those that occur in the present; a process called discounting. ${ }^{24} \mathrm{In}$ keeping with recommendations from the UK Treasury, we discounted costs at $6 \%$ per annum, and we discounted quality of life benefits at $1.5 \%$ per annum for the base case analysis. ${ }^{25}$ For information we present the results when both costs and benefits are discounted at $6 \%$.

\section{Model assumptions}

Box 3 details the assumptions made in constructing the model. The general principle maintained in these

\section{Box 3: Assumptions made in constructing model}

- A sustained effect of treatment was modelled on both progression and relapse beyond the trial's duration. Any patient who stops treatment then progresses according to rates for disease progression under conventional management but retains any benefits received at no additional cost of treatment-on the expanded disability status scale, these patients never catch up with the patients who only receive conventional management

- The effects of treatment did not deteriorate or increase over time

- The annual relative risk of all cause mortality for the multiple sclerosis cohort was assumed to be the same as a normal healthy population, minus the death observed in the London Ontario cohort

- Patients start treatment according to the Association of British Neurologist's guidelines and are treated until they reach state 7.0 or drop off treatment assumptions is to favour the novel therapies within the analysis.

In the base case scenario, patients start treatment according to the Association of British Neurologist's guidelines and are treated until they reach disability state 7.0. A 20 year time horizon is used, with patients starting treatment at 30 years of age. Costs and health benefits are discounted at $6 \%$ and $1.5 \%$, respectively.

\section{Uncertainty analysis}

We conducted multivariate Monte Carlo sensitivity analysis for uncertainty in random variables, together with scenario analysis of the management variables within the model. ${ }^{26}$ We constructed a cost effectiveness acceptability curve for the 20 year cost per QALY gained for each treatment.

We examined the impact of setting the price of all the drugs equal to the most cost effective treatment in the base case. As existing cost effectiveness analyses have adopted a wide range of time horizons, we examined the cost effectiveness as the time horizon increased between one and 20 years. ${ }^{4-9}$

\section{Results}

The primary outcome was cost per QALY gained. The price of each drug had a considerable effect on the central estimate of cost effectiveness for each drug (table 2). The use of a $6 \%$ discount rate for both costs and benefits increased the cost per QALY gained by around $75 \%$.

\section{Uncertainty analysis}

The probability that the cost effectiveness of any of the interventions is better than $£ 20000$ (\$32 250; €30 750) is in the range $3 \%$ to $18 \%$ (fig 2 ). The fact that the curve never reaches 1.0 implies that, given current evidence, all of these drugs may lead to a reduction in quality of life compared with conventional management. Figure 3 shows the cost per QALY estimated by this assessment for the treatment with the mid-range cost effectiveness as the time horizon increases from one to 20 years, together with estimates from other previous

Table 2 Cost per quality adjusted life year gained compared with conventional treatment for multiple sclerosis

\begin{tabular}{|c|c|c|c|c|c|}
\hline Intervention & Disease treated & $\begin{array}{l}\text { Base case public } \\
\text { model }(£)\end{array}$ & $\begin{array}{l}6 \% \text { discount rate } \\
\text { for costs and } \\
\text { benefits }(£)\end{array}$ & $\begin{array}{l}\text { Equal price } \\
(£ 7259)^{\star}\end{array}$ & $\begin{array}{c}\text { Base case } \\
\text { confidential model }\end{array}$ \\
\hline Interferon beta-1a 6 MIU/week (Avonex) & Relapsing remitting & 42041 & 73137 & 30473 & - \\
\hline Interferon beta-1a $22 \mu \mathrm{g} /$ week (Rebif) & Relapsing remitting & 60963 & 105718 & 44811 & - \\
\hline Interferon beta-1a $44 \mu \mathrm{g} /$ week (Rebif) & Relapsing remitting & 71732 & 124034 & 35685 & - \\
\hline Interferon beta-1b 8 MIU/week (Betaferon) & Relapsing remitting & 49664 & 86127 & 49664 & 35282 \\
\hline Glatiramer acetate $20 \mathrm{mg} /$ week (Copaxone) & Relapsing remitting & 97636 & 168539 & 108859 & - \\
\hline Interferon beta-1b 8 MIU/week (Betaferon) & $\begin{array}{l}\text { Relapsing remitting and } \\
\text { secondary progressive }\end{array}$ & 44390 & 78722 & 44390 & 39872 \\
\hline
\end{tabular}

*Price of all therapies set equal to price of therapy that was most cost effective in base case. 
assessments. The continuous line plots the cost per QALY gained for the treatment with the mid-range estimate of cost effectiveness generated by the consortium's model. A substantial proportion of the difference between estimates of cost effectiveness can be explained by the time horizon adopted in specific assessments. Almost all the previous estimates of cost effectiveness lie inside this line. For any given time horizon the replication of previous estimates of the cost effectiveness of these treatments requires the adoption of more favourable assumptions. The exceptions to this are the estimates reported by Parkin and Prosser, which are close to those produced by our model. $^{49}$

\section{Discussion}

The point estimates of the cost effectiveness of four treatments (three interferon betas and glatiramer acetate) improved considerably as the time horizon increased; those at five years were broadly comparable to previously published estimates. ${ }^{4}$ The estimates with a 20 year time horizon were markedly lower, ranging from $£ 42000$ to $£ 98000$ per QALY gained. Using the

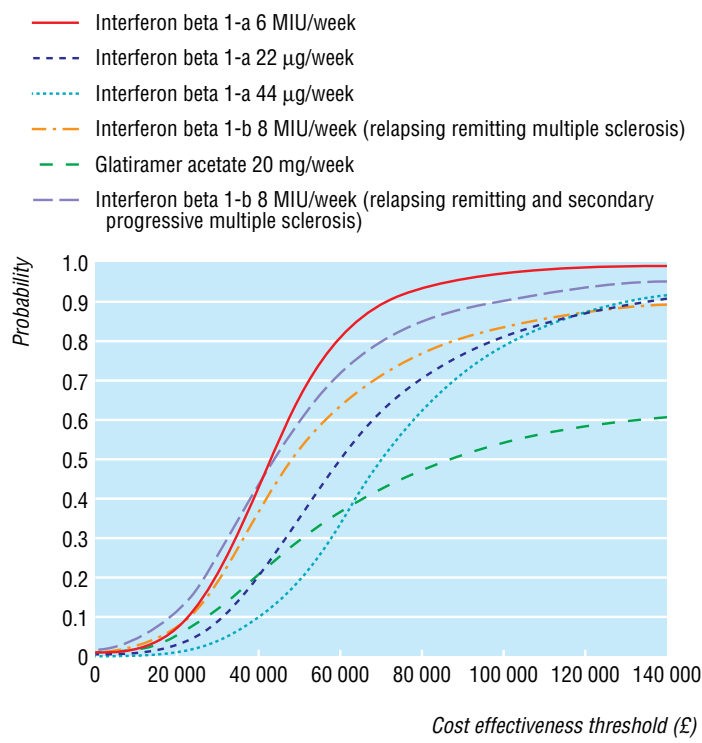

Fig 2 Cost effectiveness acceptability curves for four treatments for multiple sclerosis over 20 year time horizon

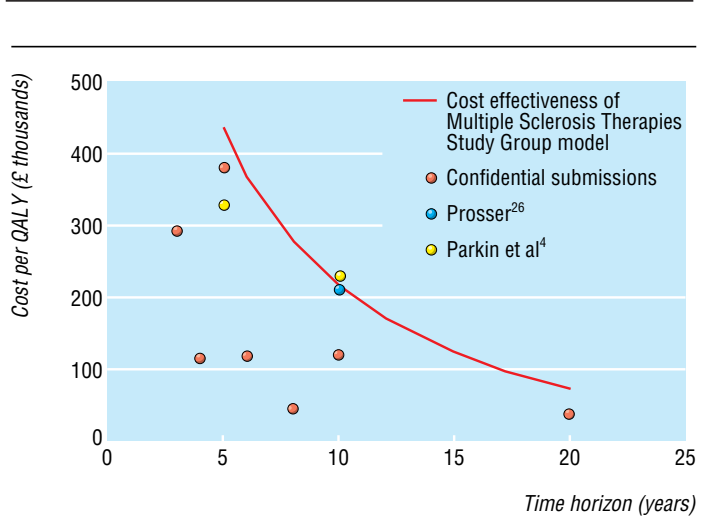

Fig 3 Impact of time horizon on cost effectiveness

\section{What is already known on this topic}

Interferon beta and glatiramer acetate are the only disease modifying therapies used to treat multiple sclerosis

Economic evaluations of these drugs have had flaws in the specification of the course of the disease, efficacy, duration of treatment, mortality, and the analysis of uncertainty

None of the existing estimates of cost effectiveness can be viewed as robust

\section{What this study adds}

The cost per quality adjusted life year gained is unlikely to be less than $£ 40000$ for interferon beta or glatiramer acetate

Experience after stopping treatment is a key determinant of the cost effectiveness of these therapies

Key factors affecting point estimates of cost effectiveness are the cost of interferon beta and glatiramer acetate, the effect of these therapies on disease progression, and the time horizon evaluated

commercial in-confidence estimates of efficacy, the most favourable estimate is $£ 35000$ per QALY and the least favourable is $£ 104000$ per QALY. These estimates did not change substantially if the treatment was assumed to start at diagnosis, rather than according to current guidance from the Association of British Neurologists, nor if patients were treated until state 10 (death). ${ }^{3}$ Care must be exercised in comparing these values, as not all the estimates of efficacy in the public domain are based on intention to treat analyses. We adopted assumptions that were favourable to treatment. The values quoted assume that the efficacy observed within the clinical trial period was maintained for as long as patients received treatment, and also that patients who stopped treatment did not catch up with those who never received treatment.

Most patients have stopped treatment by 10 years, thus the assumption we have made about disease progression after stopping treatment has a major impact on the estimate for cost effectiveness. If there is any rebound effect after stopping treatment, the cost effectiveness deteriorates.

The dataset used to estimate the costs in different states is relatively small when the patients with primary progressive and benign multiple sclerosis are removed. It is possible that the true costs are markedly different from those used in the baseline analysis, but there is no evidence to support this. The dataset used to estimate utilities for each health state is considerably larger. The utility decrement for relapse may be an underestimate as individuals experiencing severe relapses may be less likely to complete or return the questionnaire. Since the estimate may be biased towards moderate and mild relapses, we specified a wide range for the uncertainty in decrement of utility. The cost effectiveness estimates are not sensitive to this value. 
The uncertainty surrounding each of these point estimates of cost effectiveness is also large and derives primarily from the uncertainty in the actual scale of benefit gained from these interventions in terms of delayed progression of disability. Further research to establish the impact of these treatments by using robust and stable outcome measures would be of considerable value in improving the precision of estimates for cost effectiveness. ${ }^{27}$ It would also be extremely valuable to obtain real data on the progress of people once they have stopped treatment. Given the length of time that these drugs have been in use, it should be possible to gather such data. In the short term, the key modifiable determinant of the cost effectiveness of these drugs is their price.

We thank Nick Bansback (School of Health and Related Research, University of Sheffield) for his support, the stakeholders to the appraisal process, and Gisela Kobelt and colleagues for making available the costs and quality of life data. A list of the stakeholders to the appraisal process is available at www.nice.org.uk/article.asp?a $=1371$

Contributors: JC, PT, and CMcC conducted the economic modelling. KA, NJC, and $\mathrm{A}^{\prime} \mathrm{OH}$ conducted the reanalysis of confidential trial data and the London Ontario Cohort clinical course study. KC conducted a review of previous economic evaluations in multiple sclerosis. The Cost Effectiveness of Multiple Sclerosis Therapies Study Group acted as a steering group for the specification of the analysis. $\mathrm{CMcC}$ will act as guarantor for the paper.

Funding: National Institute for Clinical Excellence.

Competing interests: $\mathrm{CMcC}$ has provided consultancy services to Serono for cost effectiveness of Rebif, an interferon beta. He is employed by the University of Sheffield, under a contract funded by the UK NHS.

1 Compston A, Ebers G, Lassmann H, McDonald I, Matthews B, Wekerle H. Natural history of multiple sclerosis. McAlpine's multiple sclerosis. Edinburgh: Churchill Livingstone, 1998:191-221.

2 Richards RG, Sampson FC, Beard SM, Tappenden P. A review of the natural history and epidemiology of multiple sclerosis: implications for resource allocation and health economic models. Health Technol Assess 2002;6(10):i-73

3 Association of British Neurologists. Guidelines for treatment with interferon beta and glatiramer acetate in multiple sclerosis. London: ABN, 2001.

4 Parkin D, McNamee P, Jacoby A, Miller P, Thomas S, Bates D. A cost-utility analysis of interferon beta for multiple sclerosis. Health Technol Assess 1998;2(4):i-58.

5 Brown MG, Murray TJ, Sketris IS, Fisk JD, LeBlanc JC, Schwartz CE, et al. Cost-effectiveness of interferon beta- $1 \mathrm{~b}$ in slowing multiple sclerosis disability progression. First estimates. Int J Technol Assess Health Care 2000;16:751-67.

6 Forbes RB, Lees A, Waugh N, Swingler RJ. Population based cost utility study of interferon beta-1b in secondary progressive multiple sclerosis. BMJ 1999;319:1529-33.
7 Kendrick M, Johnson KI. Long term treatment of multiple sclerosis with interferon beta may be cost-effective. Pharmacoeconomics 2001;18:45-53.

8 National Institute for Clinical Excellence. Minutes of the technology appraisals committee meeting 13 Dec, 2000. www.nice.org.uk/article.asp?a=16091 (accessed 6 May 2001).

9 Prosser L. The cost-effectiveness of treatments for multiple sclerosis. Cambridge, MA: Harvard University, 2000.

10 Kurtzke JF. Rating neurologic impairment in multiple sclerosis: an expanded disability status scale. Neurology 1983;33:1444-52.

11 Ebers G. London Ontario cohort study: confidential data on file at National Institute for Clinical Excellence, London, 2001.

12 Weinshenker BG, Bass B, Rice GPA, Noseworthy RJ, Carriere W, Basker ville J, et al. The natural history of multiple sclerosis: a geographically based study. 1. Clinical course and disability. Brain 1989;112:133-46.

13 Patzold U, Pocklington PR. Course of multiple sclerosis: first results of a prospective study carried out on 102 MS patients from 1976-80. Acta Neurol Scand 1982;65(4):248-66.

14 IFNB Multiple Sclerosis Study Group. Interferon beta-1b in the treatment of multiple sclerosis: final outcome of the randomized controlled trial. $A m$ Acad Neurol 1995:45:1277-85.

15 PRISMS Study Group. Randomised double-blind placebo-controlled study of interferon $\beta$-1a in relapsing/remitting multiple sclerosis. Lance 1998;352:1498-504.

16 Jacobs LD, Cookfair DL, Rudick RR, Herndon RM, Richet JR, Salazir AM, et al. Intramuscular interferon beta-1a for disease progression in relapsing multiple sclerosis. Am Neurol Assoc 1996;39:285-94.

17 Johnson KP, Brooks BR, Cohen JA, Ford CC, Goldstein J, Lisak RP, et al. Copolymer 1 reduces relapse rate and improves disability in relapsing-remitting multiple sclerosis: results of a phase III multi center, double-blind, placebo-controlled trial. Am Acad Neurol 1995;45:1268-76.

18 European Study Group on interferon $\beta-1 \mathrm{~b}$ in secondary progressive MS Placebo-controlled multicentre randomised trial of interferon $\beta-1 \mathrm{~b}$ in treatment of secondary progressive multiple sclerosis. Lancet 1998:352:1491-7

19 Schering. 8 MIU Interferon $\beta$-1b clinical trial data: confidential data on file at National Institute for Clinical Excellence, London, 2001.

20 Biogen. 6 MIU Interferon $\beta$-1a clinical trial data: confidential data on file at National Institute for Clinical Excellence, London, 2001.

21 Kobelt G, Lindgren P, Parkin D, Francis DA, Johnson M, Bates D, et al. Costs and quality of life in multiple sclerosis. A cross-sectional observational study in the UK. Scandinavian Working Papers in Economics, 2000. swopec.hhs.se/hastef/papers/hastef0398.pdf (accessed 12 May 2001).

22 British Medical Association, Roval Pharmaceutical Society of Great Britain. British national formulary London: BMA, RPS, 2001:422-3. (No 42.)

23 Multiple Sclerosis Research Trust. Quality of life of people with multiple sclerosis: data on file at the National Institute for Clinical Excellence, London, 2001.

24 Lipscomb J, Weinstein MC, Torrance GW. Time preference. In: Gold MR, Siegel JE, Russell LB, Weinstein MC, eds. Cost-effectiveness in health and medicine. New York, Oxford University Press, 1996.

25 National Institute for Clinical Excellence. Guidance for manufacturers and sponsors: technology appraisals process series No 5. London: National Institute for Clinical Fxcellence, 2001.

26 Doubillet P, Begg CB, Weinstein MC, Braun P, McNeil BJ. Probabilistic sensitivity analysis using Monte Carlo simulation: a practical approach. Med Decis Making 1985;5:157-77.

27 National Institute for Clinical Excellence. Full guidance on the use of beta interferon and glatiramer acetate for the treatment of multiple sclerosis. London: NICE, 2002;1-25.

(Accepted 12 December 2002)

\section{Commentary: Evaluating disease modifying treatments in multiple sclerosis}

David H Miller

Multiple sclerosis is a chronic demyelinating disease of the central nervous system that affects one in 800 people in the United Kingdom. It usually presents in young adults, and although the course is highly variable, most people develop serious and irreversible neurological disabilities over 10 to 30 years. It has a major adverse impact on the quality of life of affected individuals and their carers. The loss of productivity and independence has important socioeconomic consequences.

Three interferon betas and glatiramer acetate are licensed as disease modifying treatments for multiple sclerosis. They are given by injection and have shown few serious adverse effects over observation periods that are currently up to 10 years. In placebo controlled trials over two years, the agents reduced the relapse rate by $30 \%$; interferon beta reduced the rate at which new lesions were detected by magnetic resonance imaging by $50-80 \%$ and modestly slowed the accumulation of disability in relapsing remitting but not secondary progressive non-relapsing multiple sclerosis. ${ }^{1}$

The use of these agents has evoked debate. Firstly, it is not known whether treatment in early relapsing
Institute of Neurology and National Hospital for Neurology and Neurosurgery, London WC1N 3BG David H Miller professor of clinical neurology d.miller@ion. ucl.ac.uk 
remitting multiple sclerosis delays the development of secondary progression and irreversible disabilitylonger term studies are needed. Secondly, the treatments are expensive, and in 2002 the National Institute for Clinical Excellence concluded that they were not cost effective. ${ }^{2}$ Chilcott et al performed the health economics analysis for the National Institute for Clinical Excellence and show that the cost per quality adjusted life year gained is high but decreases with prolonged treatment up to 20 years. This makes sense because it takes many years to develop the disabilities that account for the major costs of the disease. The model is, however, weakened by extrapolating treatment effects over longer periods than those for which data are available, using a single study of the clinical course of the disease to derive disease progression rates, and by the unpredictability of the disease and the difficulty in capturing all aspects of its impact on patients.

In the face of such uncertainties, the Department of Health has introduced a scheme for providing disease modifying treatments in the NHS. This risk sharing scheme provides treatment for ambulant patients with clinically active relapsing disease, ${ }^{3}$ with annual monitoring of neurological status over 10 years, calculation of cost effectiveness based on actual long term data, and adjustment of the cost of the drugs (if required) to make them cost effective. The scheme has important scientific and practical limitations and will need substantial investment to generate reliable data. Nevertheless, it is a constructive approach in addressing a difficult problem, and it is hard to see a realistic alternative. Providing no disease modifying treatments to patients with frequent and disabling relapses would seem unreasonable, and a long term placebo controlled trial is unlikely to be acceptable to many patients or neurologists.

Research is needed to identify those who will benefit most from disease modifying treatments. This may be possible by independent meta-analysis of large datasets from clinical and magnetic resonance imaging studies derived from multiple trials and natural history studies of the clinical course of multiple sclerosis. ${ }^{4}$ Work must also go on to develop more effective treatments.

Competing interests: DHM has received grants from Elan, Biogen, and Schering, honoraria for giving expert advice to Biogen, Schering, Wyeth, and Bristol Myers Squibb, and lecture fees from Serono.

1 Rice GPA, Incorvaia B, Munari L, Ebers G, Polman C, D'Amico R, et al. Interferon in relapsing-remitting multiple sclerosis. Cochrane Library. Issue 4. Oxford: Update Software, 2001.

2 National Institute for Clinical Excellence. Beta interferon and glatiramer acetate for the treatment of multiple sclerosis. Technology appraisal guidance. www.nice.org.uk [No 32, Jan 2002.]

3 Association of British Neurologists. Guidelines for the use of beta interferons and glatiramer acetate in multiple sclerosis. Jan 2001. www.theabn.org

4 Noseworthy J, Kappos L, Daumer M. Competing interests in multiple sclerosis research. Lancet 2003;361:350-1. 